\title{
Parallel proteomic analysis in muscle-invasive bladder transitional cell carcinoma and cancer-related stroma
}

\author{
H.T. Niu ${ }^{1 *}$, X.J. Qi" ${ }^{2 *}$, Y.Q. Liu ${ }^{3}$, Y.W. Cao ${ }^{1}$, Q. Dong ${ }^{4}$ and X.S. Wang ${ }^{1}$ \\ ${ }^{1}$ Department of Urology, Medical College, Affiliated Hospital, \\ Qingdao University, Qingdao, Shandong Province, China \\ ${ }^{2}$ Department of Urology, People's Hospital of Linzi District, Zibo, \\ Shandong Province, China \\ ${ }^{3}$ Department of Clinical Laboratory, People's Hospital of Hezhou City, \\ Hezhou, Guangxi Province, China \\ ${ }^{4}$ Department of Pediatric Surgery, Medical College, Affiliated Hospital, \\ Qingdao University, Qingdao, Shandong Province, China \\ *These authors contributed equally to this study. \\ Corresponding author: X.S. Wang \\ E-mail: qyxinshengwang@126.com
}

Genet. Mol. Res. 12 (4): 4251-4263 (2013)

Received August 6, 2012

Accepted November 22, 2012

Published February 28, 2013

DOI http://dx.doi.org/10.4238/2013.February.28.28

\begin{abstract}
To bring about improvements in cancer biology research and elucidate mechanism-based therapeutic targets, we studied the proteome expression profile of purified normal urothelial cells (cancer cells) and normal stromal cells (cancerous stromal cells). Based on the expression profile, biomarker discovery and the mechanisms of multistep carcinogenesis were explored. We found that 1412/1403 unique proteins commonly appeared in 4 sets of paired cancer/normal tissue, and 1753 proteins were differentially expressed. Three hundred and forty-one proteins were repeatedly expressed in both cancer and cancer stromal cells; 358 proteins were repeatedly expressed in both normal urothelial and normal stromal cells. Among them, 186/203 proteins were specific repeat
\end{abstract}


expressions in cancer/normal tissue and thought to play an important role in cancer-stroma interactions. Differential proteins were further analyzed using bioinformatic tools and compared with the published literature. GO enrichment/depletion analysis indicated that carcinogenesis involved all the biological processes and all the cellular components. Five hundred and sixty-eight differential proteins were located in the well-known biological Kyoto Encyclopedia of Genes and Genomes pathways, including metabolic pathways, ribosome spliceosome, and endocytosis. One hundred and thirty-nine of the 186 proteins that displayed specific repeat expressions in cancer tissue were located in the biological Kyoto Encyclopedia of Genes and Genomes pathways and are thought to be candidate biomarkers for targeted therapy.

Key words: Muscle-invasive bladder cancer; Stroma; Biomarker; Pathway analysis

\section{INTRODUCTION}

Despite the elaborate characterization of risk factors, muscle-invasive bladder transitional cell carcinoma (BTCC) is still a major epidemiological problem with an incidence that continues to rise annually (Jemal et al., 2010). The standard therapeutic methods of muscleinvasive BTCC are radical cystectomy (RC) followed by postoperative care. Although much progress has been made in surgical techniques and perioperative chemoradiation, the 5-year disease-specific survival after RC remains at 50-60\% (Niu et al., 2008). Advances in molecular biology during the last decade have yielded reports of a large number of cancer-related molecules (Schiffer et al., 2009; Pezaro et al., 2012). However, the carcinogenic mechanism is still unclear (Kaufman et al., 2009).

Bladder cancers are heterogenous cellular entities composed of epithelial cells and cells of the stroma in which they reside. A reciprocal dynamic interaction occurs between cancer cells and the surrounding stroma under certain physiological and pathological conditions. This tumor-host communication interface mediates all biological behaviors of bladder cancer (Wooster, 2012). Compared with neoplastic epithelial cells, cancerous stroma is more complex, and separating the stromal components one by one is difficult. However, the protein expression profile of cancerous stroma reflects the final outcome of the complex and dynamic interaction of all kinds of stromal cells. The discovery of proteome expression profiles that are integral to either neoplastic cells or their specialized stroma may advance our understanding of the biology of muscle-invasive BTCC and yield novel biomarkers and targets for anticancer therapies.

In this study, laser capture microdissection (LCM) was used to harvest pure subpopulation cells. Using LCM, we subdivided the clinical bladder cancer specimens into normal urothelial cells (cancer cells)/normal stromal cells (cancerous stromal cells). Next, two-dimensional liquid chromatography-tandem mass spectrometry (2-D-LC-MS/MS) - namely, a shotgun strategy for the direct analysis of complex mixtures of peptides - was used to identify the expression profiles. Based on the expression profile of subpopulation cells, neoplastic cells and corresponding stroma were investigated separately and systematically.

Until now, many of the answers to questions about tumor-stroma interaction lay in 
the identity of ligands, receptors, and effectors of signaling patterns expressed by stroma and cancer cells, but the explanation of the complex network was far from solved (Roth and Czernobilsky, 2011). In present study, we hypothesized that the co-expressed proteins in both cancer and stromal cells control tumor-stroma interaction, and we deduced corresponding pathway changes to explain the cross-talk between these cells. After data comparison and deep analysis, we present several candidate biomarkers with which to understand cancer biology as well as effective targets for anticancer therapeutics.

\section{MATERIAL AND METHODS}

\section{Patients and tissue samples}

A total of 4 paired muscle-invasive BTCC and normal urothelial samples (confirmed by 2 individual pathological diagnoses) were obtained from patients treated at the Affiliated Hospital of Medical College, Qingdao University, immediately after RC treatment for primary muscle-invasive BTCC. No patient had distant metastatic disease at cystectomy and no patient presented with carcinoma in situ. The tumor and the adjacent (5-cm margin) microscopically normal urothelial samples were rinsed in sterile phosphate-buffered saline and snap frozen in liquid nitrogen within $30 \mathrm{~min}$ of removal. The research protocol was approved by the institutional review board, and informed consent was obtained from patients.

\section{LCM}

LCM was performed with a Leica AS LMD (Sunnyvale, CA, USA) system. Sections $(8 \mu \mathrm{m})$ of freshly prepared tissues were stained with hematoxylin and eosin using standard manufacturer protocols. The stained sections were air dried and then subjected to LCM, and approximately 500,000 normal urothelial cells (cancer cells)/normal stromal cells (cancerous stromal cells) were laser captured with a $15-\mu \mathrm{m}$ laser spot using multiple captures. To avoid the degradation of protein, we examined the cells within $120 \mathrm{~min}$ of each capture. Each cell population was determined to be $95 \%$ homogeneous through microscopic visualization of the captured cells (Figure 1). Microdissected cells were dissolved in lysis buffer [95 mM urea, 4\% 3-((3-cholamidopropyl)-dimethylammonio) propanesulfonate, $40 \mathrm{mM}$ Tris, $65 \mathrm{mM}$ dithiothreitol]. Samples were solubilized via sonication using 20 -s bursts followed by ice cooling (20 s) in a process that was repeated 5 times. Then the crude tissue extracts were centrifuged for 45 min at 15,000 rpm to remove the undissolved pellets. The protein concentrations of samples were measured with a Bio-Rad protein assay kit. All samples were stored at $-80^{\circ} \mathrm{C}$ until use.

\section{Digestion of protein mixture and 2-D-LC-MS/MS}

Samples prepared using LCM technology were deposited in precipitation solution ( $50 \%$ acetone $/ 50 \%$ ethanol $/ 0.1 \%$ acetic acid, sample volume:precipitation solution volume $=1: 5$ ) for at least $12 \mathrm{~h}$ at $-20^{\circ} \mathrm{C}$. The pellets were washed with $100 \%$ acetone and $70 \%$ ethanol and redissolved in $6 \mathrm{mM}$ guanidine- $\mathrm{HCl} / 100 \mathrm{mM}$ Tris, $\mathrm{pH}$ 8.3. The concentrations were measured with a Bio-Rad protein assay kit. Next, $250 \mu \mathrm{g}$ soluble proteins were reduced with dithiothreitol (final concentration, $20 \mathrm{mM}$ ) and subsequently alkylated with iodoacetamide 
(final concentration, $40 \mathrm{mM}$ ). After desalting and stain removal via ultrafiltration with a Microcon-10, the protein mixture was incubated overnight at $37^{\circ} \mathrm{C}$ with trypsin (trypsin:protein mixture $=1: 30 \mathrm{w} / \mathrm{w}$ ). Two-dimensional high-performance LC separations were performed on a ProteomeX workstation equipped with 2 capillary LC pumps (ThermoFinnigan, CA, USA). The flow rate of both the salt and the 4 analytical pumps was $120 \mu \mathrm{L} / \mathrm{min}$ and was approximately $1.5 \mu \mathrm{L} / \mathrm{min}$ after the split. Nine salt concentration ranges $-0,25,50,75,100,150,200$, 400 , and $800 \mathrm{mM}$ ammonium chloride - were used for a step gradient. The mobile phases used for the reverse phase were $\mathrm{A}, 0.1 \%$ formic acid in water, $\mathrm{pH} 3.0$; and $\mathrm{B}, 0.1 \%$ formic acid in acetonitrile. A Finnigan LTQ linear ion trap mass spectrometer was used for the MS/MS experiment with an ion transfer capillary of $160^{\circ} \mathrm{C}$ and spray voltage of $3.0 \mathrm{kV}$. Normalized collision energy was $35.0 \%$.

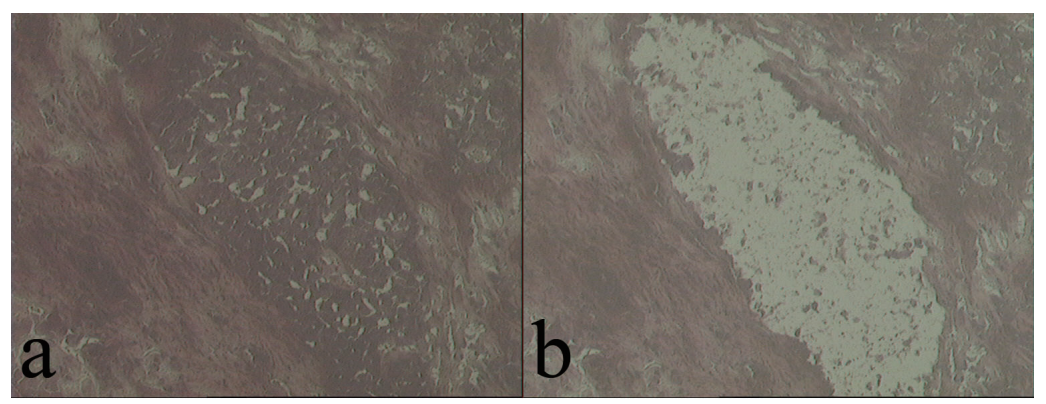

Figure 1. Harvest purify cancer cells by laser capture microdissection (LCM). (a) cancer sample before LCM; (b) residual tissue after removal of cancer cells by LCM.

After acquisition of full-scan mass spectra, $10 \mathrm{MS} / \mathrm{MS}$ scans were acquired for the next 10 most intense ions using dynamic exclusion. Peptides and proteins were identified with the Sequest software (ThermoFinnigan), which uses the MS and MS/MS spectra of peptide ions to search against the publicly available International Protein Index (IPI) database. The spectra for singly charged peptides with a cross-correlation score to a tryptic peptide (Xcorrs) greater than 1.9, the spectra for doubly charged tryptic peptides with Xcorrs of at least 2.2, and the spectra for triply charged tryptic peptides with Xcorrs above 3.75 were accepted as correctly identified according to the Sequest software. For all spectra analyzed, $\Delta C n$ values were above 0.1 .

\section{GO biological process analysis}

The normal/cancer tissue-specific proteins were categorized as GO assignments (www.geneontology.org), and the GOfact software was used to find statistically over- or underrepresented GO categories in biological data as the tool for enrichment analysis of our proteome dataset (Li et al., 2005). For enrichment analysis, a test dataset (our identified proteins) and a reference set of GO annotation for the complete human proteome were obtained. Per instructions on the GOfact webpage, the custom GO annotation for the reference set (the whole IPI human dataset) was created by extracting the GO annotations available for human IPI IDs from the EBI GOA Human 80.0 release. The analysis was carried out using a "hyper 
geometric test"; GO terms with $\mathrm{P}<0.05$ or $\mathrm{P}<0.01$ were selected as enriched/depleted or significantly enriched/depleted.

\section{Pathway analysis}

To create an overview of our comparative proteomics analysis, we first categorized the normal urothelial cells/cancer cells and normal stromal cells/cancer stromal cell-specific proteins. Next, the IPI names of the differentially expressed proteins were converted to SWISS-PROT names for SWISS-PROT, a protein sequence database of low redundancy with high levels of annotation. The ArrayTrack software was used for pathway analysis (Jefferson, AK, USA). ArrayTrack offers a simple query interface with which to retrieve information about human protein expression profiles and provides direct connections to related metabolic and regulatory pathways available from the Kyoto Encyclopedia of Genes and Genomes (KEGG) (Tong et al., 2004). Most important, ArrayTrack software can analyze expression profiles without consideration of differential abundance. For statistical analysis, a $\mathrm{P}$ value for pathway enrichment was obtained using the hypergeometric test, and $\mathrm{P}<0.05$ was considered to be statistically significant.

\section{RESULTS}

\section{Identification of proteins}

We found that $885 / 890$ proteins commonly appeared in 4 paired cancer/normal urothelial samples, and 868/872 proteins commonly appeared in 4 paired cancerous stromal/normal stromal samples. The whole expression profile was considered as the sum of commonly appearing proteins in each cancerous or normal tissue. For example, the sum of the proteins identified in cancer cells and cancerous stromal cells was considered as the whole cancer expression profile. The number of proteins identified from each sample is listed in Table 1. To reveal the general biological characteristics of the cancer and to reduce the complexity of the data, we minimized the heterogeneity by analyzing only the commonly appearing proteins in each pair of samples. After comparison, 341 proteins were repeatedly expressed in both cancer and cancer stromal cells, and 358 proteins were repeatedly expressed in both normal urothelial and normal stromal cells. After duplicate data were eliminated, 1412/1403 unique proteins commonly appeared in 4 paired cancer/normal tissue samples.

\begin{tabular}{|c|c|c|c|c|c|c|c|}
\hline \multirow[t]{2}{*}{ Specimen } & \multicolumn{2}{|c|}{ Cancer tissue } & \multirow[t]{2}{*}{ Repeat expression* } & \multicolumn{2}{|c|}{ Normal tissue } & \multirow[t]{2}{*}{ Repeat expression** } & \multirow[t]{2}{*}{ Overlapping expression*** } \\
\hline & Cancer & Stroma & & Normal & Stroma & & \\
\hline 1 & 990 & 998 & 341 & 983 & 982 & 358 & 155 \\
\hline 2 & 976 & 991 & & 1032 & 964 & & \\
\hline 3 & 996 & 957 & & 1058 & 1022 & & \\
\hline 4 & 1036 & 1086 & & 1011 & 979 & & \\
\hline
\end{tabular}

*Repeat expression proteins in cancer cells and cancer stromal cells. ${ }^{* *}$ Repeat expression proteins in normal urothelial and normal stromal cells. *** Overlapping expression proteins in both cancer tissue and normal tissue. 
A total of 1753 proteins were differentially expressed between cancer and normal tissue. Among them, 883 proteins were specifically expressed in cancer tissue, and 870 proteins were specifically expressed in normal tissue. Of the $341 / 358$ repeatedly expressed proteins, 155 proteins displayed overlapping expression in either cancer or normal tissue, and 186/203 proteins displayed specifically repeated expression in cancer/normal tissue. The IPI names of the specific repeat expression proteins were converted to SWISS-PROT names and are listed in Table 2.

Table 2. Specific repeat expression proteins in cancer/normal tissue.

\begin{tabular}{|c|c|}
\hline Specific repeat expression in cancer tissue & Specific repeat expression in normal tissue \\
\hline $\begin{array}{l}C C D C 6, R B P M S, P I G R, C R K, \mathrm{AK} 1 \mathrm{C} 2, \mathrm{RD} 23 \mathrm{~B}, \\
\text { LIMA1, DD19B, SF13A, SFRS3, CBX1, HNRDL, } \\
\text { SFRS9, RS20, LSP1, TBB3, QCR1, HNRH1, } \\
\text { C1QBP, IF4H, TEBP, RAP1B, RAB5C, RUXG, } \\
\text { GSHR, VTA1, SIOOP, ACON, SMD2, LYSC, HDGF, } \\
\text { COX5B, CYTB, ECHB, PIP, PDCD5, PRS8, VDAC2, } \\
\text { CPNE3, ATPD, CPNS1, DSG1, FAS, RSMB, RHOC, } \\
\text { G6PI, TCPZ, PLIN1, ACTZ, DCTN1, SRC8,ERH, } \\
\text { SNX3, SSBP, SEP15, TENA, RL9, ANT3, S10A4, } \\
\text { CYTA, AT8A1, ACSM1, DBNL, PSA3, HNRPM, } \\
\text { GRN, FABP4, RS19, CTNA1, ROA1, CAH1, TBCA, } \\
\text { ILF3, PEBP1, S10A7, PRDX6, CH10, QCR7, } \\
\text { RUXF, LGUL, RS16, IMDH2, RAB14, HCDH, } \\
\text { FBLN5, NUCB1, LAMB2, F13A, CBX3, CO1A1, } \\
\text { SYUG, LAMC1, FIBB, PARK7, APOH, TRFL, } \\
\text { TLN1, PA2G4, BASP1, PDIA6, PSMD1, MED8, } \\
\text { ELOC, TRA2B, FLNA, ATPB, APOA4, CO6A2, } \\
\text { HSP71, ITIH2, K2C7, FBN1, NB5R3, LAMA4, } \\
\text { ANXA5, ROAA, IF5A1, HS90A, HV304, ROA2, } \\
\text { MYH10, HBA, VIME, PPIA, CNBP, TIF1B, ATPA, } \\
\text { K1C17, H4, ANXA2, ENOA, LEG3, ALDOA, HBD, } \\
\text { RINI, TAGL2, A1AT, UBA1, PPIB }\end{array}$ & $\begin{array}{l}\text { GLYR1, BAG2, AIFM1, FERM2, LASP1, HYOU1, } \\
\text { TRIM } 7, \text { TIM13, ANXA7, SRBS1, FKBP2, GDIR2, } \\
\text { ZO2, AT2A3, PYGB, PDLI3, B2MG, 2DRA, 2B18, } \\
\text { TINAL, SPTB2, PFD6, PP1G, SPTC1, SDPR, CRIP2, } \\
\text { PERE, PERM, RPB4, ACINU, ATPO, HSP72, } \\
\text { TBA4A, POSTN, RS5, PABP1, RLA1, MAP1B, } \\
\text { CSPG2, CBPA3, METK2, TRYA1, TCPE, ETFA, } \\
\text { CLIC1, IDHP, CATD, CD59, PRDX4, HNRPR, } \\
\text { PGS2, SAP, EMIL2, EMIL1, HNRH3, TPM2, } \\
\text { PGM5, EIF1, RHG07, RAB10, DHE3, EHD1, } \\
\text { CERU, HCD2, HNRPQ, H2AV, RNAS2, LPAR6, } \\
\text { S22AB, RHG01, HXA4, PRELP, CO3A1, CNN1, } \\
\text { ACTS, C4BPA, RS6, APOC2, VIGLN, APOB, } \\
\text { CAP7, SODM, HRG, TERA, A1BG, PDLI7, } \\
\text { NALD2, TBB4, LG3BP, LPP, CLH1, ANXA3, } \\
\text { ECHM, TENX, SH3L1, MIME, IF4A1, AN32A, } \\
\text { CAPZB, TRIM9, EFTU, CANB1, MMP9, ELNE, } \\
\text { SODE, PSB10, CATG, HNRPD, NID2, CY1, } \\
\text { FBLN3, DSCAM, CFAH, LAP2B, SYFA, SERPH, } \\
\text { KNG1, AL2SA, PP14A, H2AY, RPESP, ZBT45, } \\
\text { GT2D1, SET, EHD2, AL1B1, SSH1, SND1, } \\
\text { SMRC2, WDR82, CP011, LAC, ZA2G, TXND5, } \\
\text { COEA1, FLNC, F193A, MICA2, S10AA, TPM3, } \\
\text { ATS16, EF2, PLEC1, APEX1, 1433F, ITIH4, C1TC, } \\
\text { CY24B, TPM2, HMGB2, RL22, GSTK1, COPB2, } \\
\text { AK1A1, TPM2, KU86, ICAL, HSPB7, ANXA6, } \\
\text { GBG12, MYLK, MACF1, DPYL2, ROS, TUT4, } \\
\text { CLUS, IC1, PALLD, ITIH1, SNAG, COFA1, CATB }\end{array}$ \\
\hline
\end{tabular}

Italic indicates that the proteins have been previously reported in relation to cancer.

\section{GO and GO enrichment/depletion analysis}

Of the $883 / 870$ proteins that were specifically expressed in cancer/normal cells, 556/534 owned biological process annotation and 606/582 owned cellular component annotation. Compared with the entire list of the IPI (IPI_Human, versions 3.53; 219,486 entries for 30,786 proteins), 46/49 GO terms exhibited as enriched and 21/16 exhibited as depleted in biological processes and 47/45 exhibited as enriched and 4/6 exhibited as depleted in cellular components. Figures 2 and 3 show the enrichment/depletion analyses of biological processes and cellular components. Eighteen of 15 proteins belonged to the term cell proliferation and 20/14 belonged to the term ribosome in tumor/normal tissue (Table 3). 


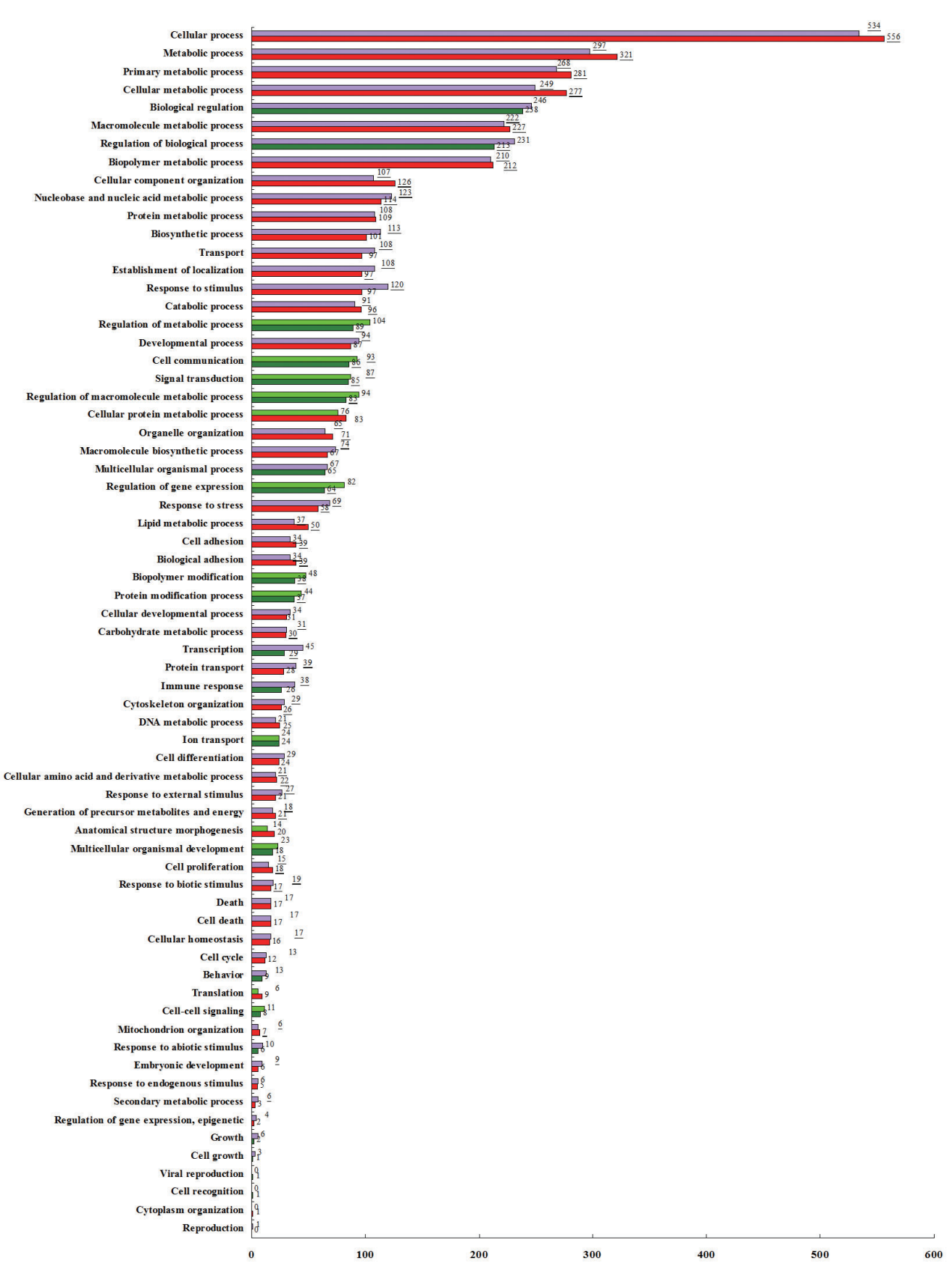

Figure 2. Enriched/depleted GO biological terms of the cancer/normal-specific expressed profile. Red/purple indicate enriched terms in cancer/normal tissue; dark green/light green indicate depleted terms in cancer/normal tissue. Underline indicates significantly enriched/depleted terms. 


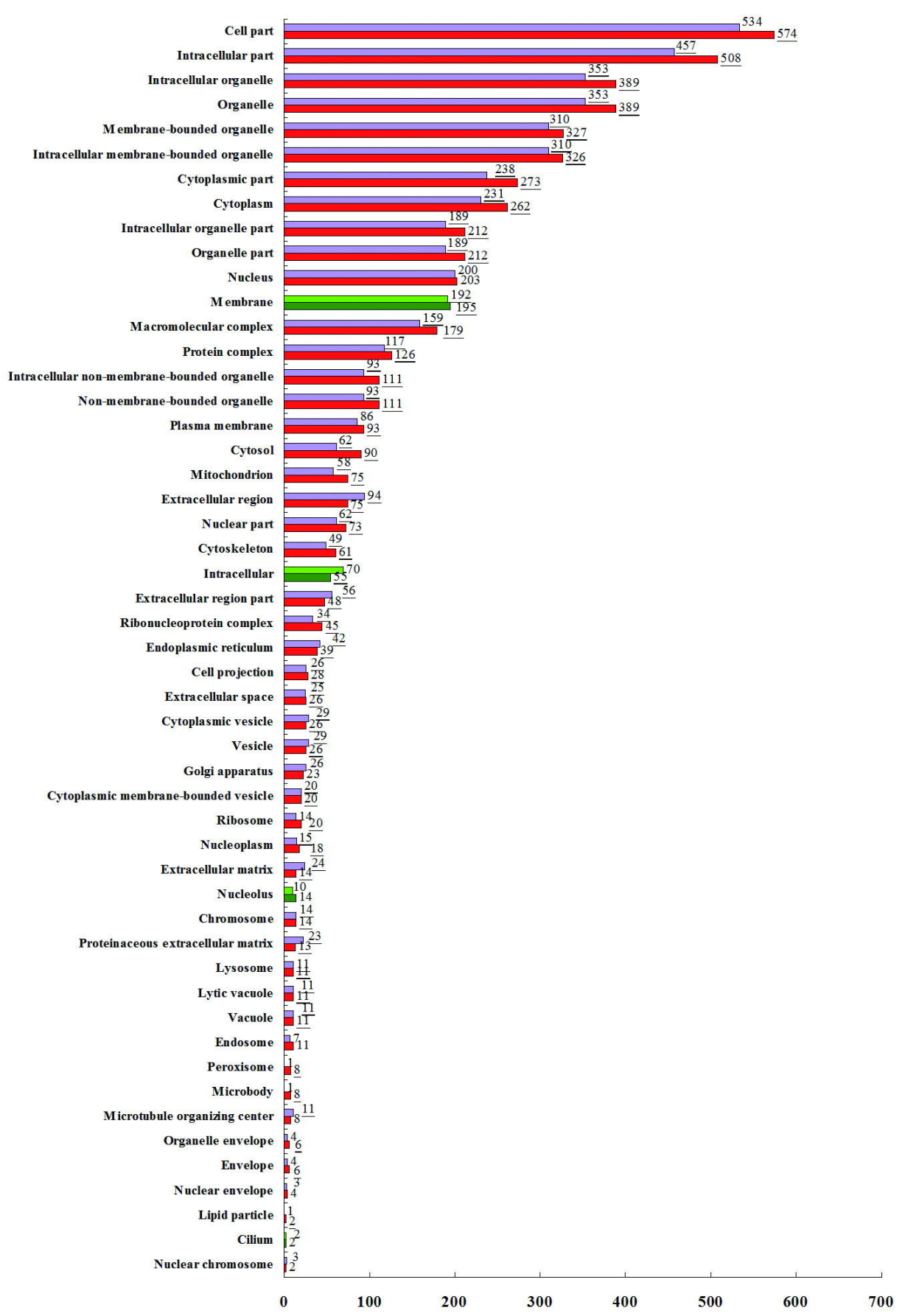

Figure 3. Enriched/depleted GO cellular terms of the cancer/normal-specific expressed profile. Red/purple indicate enriched terms in cancer/normal tissue; dark green/light green indicate depleted terms in cancer/normal tissue. Underline indicates significantly enriched/depleted terms. 
Table 3. Proteins that belong to the term of cell proliferation and ribosome by GO enrichment/depletion analysis.

\begin{tabular}{lll}
\hline Tissue & Cell proliferation & Ribosome \\
\hline Cancer & PESC, PEDF, IF5A2, KHDR1, HELLS, PDXK, & RS5, RS10, RLA0, RS20, RS25, RM49, RS12, RL23A, RS6, RL9, \\
& DP13A, HDGF, CXCL7, XPO2, S10A6, MET, & RS19, RL38, APEX1, RS4X, RS16, RS17, RL21, RL7A RL3 \\
\multirow{2}{*}{ Normal } & B1AL80, FSCN1, IMDH2, PA2G4, MYH10, & \\
& CSRP2, IF16, TPX2, MARE1, UBR5, B9A6J0, & GCN1L, RS11, RLA1, RL23, RS20, RL19, RS24, APEX2, RS8, \\
& DNL14, IMDH2, PA2G4, S100B, ZEB1, MYH10, NUDC & RL22, RS1, RL10A, RM14 \\
\hline
\end{tabular}

\section{Significant pathways and identification of potential biomarkers}

We used the ArrayTrack software to analyze 1753 differentially expressed proteins as well as the 186 proteins that were specifically repeatedly expressed in cancer tissue. After analysis, 568 differential proteins were found in the well-known biological KEGG pathways, including metabolic pathways, ribosome, spliceosome, endocytosis, and others. These pathways may have decisive roles in the multi-step carcinogenesis and development of muscleinvasive BTCC. Table 4 lists the major altered pathways that include at least 20 differentially expressed proteins. One hundred thirty-nine of the 186 proteins with specifically repeated expression in cancer tissue were located in the biological KEGG pathways. Table 5 lists the major altered pathways that include at least 6 specifically repeatedly expressed proteins.

\begin{tabular}{|c|c|}
\hline Pathway (category) & SWISS-PROT name \\
\hline $\begin{array}{l}\text { Metabolic pathways } \\
\text { (unknown) }\end{array}$ & 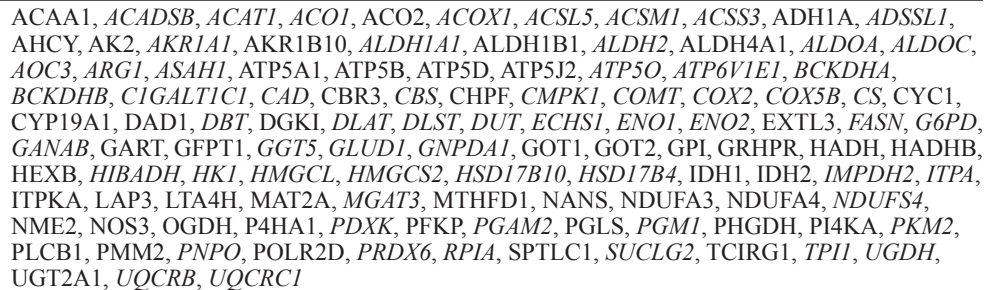 \\
\hline $\begin{array}{l}\text { Systemic lupus erythematosus } \\
\text { (Immune disorders) }\end{array}$ & $\begin{array}{l}\text { C8G, C9, CTSG, ELANE, H2AFV, H2AFY, H3F3A, H3F3B, HIST1H2AA, HIST1H2BA, } \\
\text { HIST1H4A, HIST1H4B, HIST1H4C, HIST1H4D, HIST1H4E, HIST1H4F, HIST1H4H, HIST1H4I, } \\
\text { HIST1H4J, HIST1H4K, HIST1H4L, HIST2H3A, HIST2H3C, HIST2H3D, HIST2H4A, HIST2H4B, } \\
\text { HIST4H4, HLA-DRA, HLA-DRB1, SNRPB, SSB, TNF }\end{array}$ \\
\hline $\begin{array}{l}\text { Spliceosome } \\
\text { (Transcription) }\end{array}$ & $\begin{array}{l}\text { ACIN1, CRNKL1, EIF4A3, HNRNPA1, HNRNPM, HSPA1A, HSPA1B, HSPA2, HSPA6, LSM3, } \\
N H P 2 L 1, \text { PRPF8, RBM8A, RBMX, SFRS13A, SFRS2, SFRS3, SFRS4, SFRS5, SFRS6, SFRS9, } \\
N R N P 200, \text { SNRPB, SNRPC, SNRPD2, SNRPF, SNRPG, TRA2A, TRA2B, U2AF2 }\end{array}$ \\
\hline $\begin{array}{l}\text { Focal adhesion } \\
\text { (Cell communication) }\end{array}$ & $\begin{array}{l}\text { CAPN2, CAV1, CDC42, COL1A1, COL3A1, COL4A2, COL6A2, CRK, CTNNB1, DOCK1, EGFR, } \\
F L N A, \text { FLNC, FYN, ILK, ITGA5, ITGA6, ITGB4, LAMA4, LAMB2, LAMC1, MET, MYLK, } \\
\text { PIK3R3, PPP1CC, FAK, PRKCG, RAC2, RAP1B, ROCK2, TLN1, TLN2, TNC, TNXB, VWF }\end{array}$ \\
\hline $\begin{array}{l}\text { Huntington's disease } \\
\text { (Neurodegenerative diseases) }\end{array}$ & $\begin{array}{l}\text { ATP5A1, ATP5B, ATP5D, ATP5O, CASP } 8, \text { CLTA, CLTB, CLTC, CLTCL1, COX2, COX5B, } \\
C O X 7 A 2, \text { CYC1, DCTN1, DLG4, DNAH2, EP300, NDUFA3, NDUFA } 4, N D U F S 4, \text { PLCB1, } \\
\text { POLR2D, RCOR1, SLC25A5, SOD2, TAF4, UQCRB, UQCRC1, VDAC2 }\end{array}$ \\
\hline $\begin{array}{l}\text { Alzheimer's disease } \\
\text { (Neurodegenerative diseases) }\end{array}$ & $\begin{array}{l}\text { APOE, } A T P 2 A 2, \text { ATP2A3, ATP5A1, ATP5B, ATP5D, ATP5O, CAPN1, CAPN2, CASP8, COX2, } \\
C O X 5 B, C O X 7 A 2, \text { CYC1, GRIN2C, HSD17B10, NDUFA3, NDUFA4, NDUFS4, PLCB1, PPP3R1, } \\
T N F, U Q C R B, U Q C R C 1\end{array}$ \\
\hline $\begin{array}{l}\text { Endocytosis } \\
\text { (Transport and catabolism) }\end{array}$ & $\begin{array}{l}A C A P 2, \mathrm{ARAP} 3, \mathrm{CDC} 42, C H M P 5, \text { CLTA, CLTB, CLTC, CLTCL1, DNM2, EEA1, EGFR, EHD1, } \\
\text { EHD2, EHD4, FAM125B, HLA-A, HLA-B, HLA-C, HSPA1A, HSPA1B, HSPA2, HSPA6, MET, } \\
\text { RAB11FIP3, RAB5C, VTA1 }\end{array}$ \\
\hline $\begin{array}{l}\text { Regulation of actin cytoskeleton } \\
\text { (Cell motility) }\end{array}$ & $\begin{array}{l}A R P C 1 B, \mathrm{ARPC} 2, A R P C 3, \text { ARPC5, CDC42, CFL2, } C R K, \text { CYFIP1, DOCK1, EGFR, ENAH, } F 2 \text {, } \\
\text { GNG12, IQGAP3, ITGA5, ITGA6, ITGB4, MYH10, MYH14, MYLK, PIK3R3, PPPICC, PLXNB1, } \\
\text { RAC2, ROCK, RRAS2, SSH1, SSH3, WASF2 }\end{array}$ \\
\hline $\begin{array}{l}\text { Ribosome } \\
\text { (Translation) }\end{array}$ & $\begin{array}{l}\text { RPL10A, RPL19, RPL21, RPL22, RPL23, RPL23A, RPL38, RPL3, RPL7A, RPL9, RPLP0, RPLP1, } \\
\text { RPS10, RPS12, RPS16, RPS17, RPS19, RPS20, RPS25, RPS4X, RPS5, RPS6, RPS8 }\end{array}$ \\
\hline
\end{tabular}

Italic indicates that the proteins are specific expressed in cancer specimen. 


\begin{tabular}{lll}
\multicolumn{2}{c}{ Table 5. Pathway analysis on the specific repeat expressed proteins in cancer tissue. } \\
\hline Pathway & Proteins & Category \\
\hline Systemic lupus erythematosus & HIST1H4A, HIST1H4B, HIST1H4C, HIST1H4D, & Immune disorders \\
& HIST1H4E, HIST1H4F, HIST1H4H, HIST1H4I, & \\
& HIST1H4J, HIST1H4K, HIST1H4L, HIST2H4A, & \\
HIST2H4B, HIST4H4, SNRPB & Transcription/genetic information processing \\
Spliceosome & HNRNPA1, HNRNPM, HSPA1A, HSPA1B, SFRS13A, & \\
Parkinson's disease & SFRS3, SFRS9, SNRPB, SNRPD2, SNRPF, SNRPG, TRA2B & Neurodegenerative diseases \\
Focal adhesion & ATP5A1, ATP5B, ATP5D, COX5B, PARK7, UBA1, & \\
ECM-receptor interaction & COL1A1, COL6A2, CRK, FLNA, LAMA4, LAMB2, LAMC1, & Cell communication \\
Huntington's disease & RAP1B, TLN1, TNC & Signaling molecules and interaction \\
Oxidative phosphorylation & ATP5A1, COL6A2, LAMA4, LAMB2, LAMC1, TNC & Neurodegenerative diseases \\
\hline & UQCRC1, VDAC2 2 COX5B, DCTN1, UQCRB, & Energy metabolism \\
\hline
\end{tabular}

\section{DISCUSSION}

Based on our preliminary study in which we separately identified the expression profiles of neoplastic cells and stromal cells, LCM was used as the initial step in the present research. 2-D-LC-MS/MS technology was then used to construct the proteome expression profiles of subpopulation cells. Although relative quantitative information cannot be obtained with these methods, a specifically expressed protein is one that is either expressed in only 1 tissue or displays significant differences in expression between the same sample amounts. With these advantageous technologies, we comprehensively and separately compared the expression profile within paired samples. After the comparison, we found that 1412/1403 proteins commonly appeared in 4 paired cancer/normal specimens, and 1753 proteins showed differential expression. Further analysis showed that 568 differential proteins occurred in the well-known biological KEGG pathways and were potential biomarkers. Several biomarker candidates originating from this expression profile have been reported. For example, caveolin-1 promotes metastasis of bladder cancer through a pharmacologically tractable common downstream signaling pathway and has been further researched for personalized therapy to suppress metastases (Thomas et al., 2011). Talin1 overexpression enhances cancer cell adhesion, migration, and invasion by activating survival signals and conferring resistance to anoikis (Sakamoto et al., 2010). Other examples are adenylate cyclase-associated protein 1 and human fatty acid synthase (Yamazaki et al., 2009; Shao et al., 2010).

In the context of all human proteins deposited in the IPI, the enrichment/depletion of the functional classes means that the proteins in these categories are over- or underrepresented in this profile and reflect the biologically specific categories of these data. With the advantage of wide proteome coverage and GO analysis, our outcome indicates that the global over- or underrepresented terms in biological processes and cellular components of cancer/normal tissue were quite consistent. Figures 2 and 3 show the subtlety of the global changes in the comparison between cancer and normal tissue. Beyond this global consistency, the proteins clustered in each term were completely different (see Table 3 ). The various proteins clustered in each GO term reflect that different molecular networks can maintain the same basic cell functions and behaviors, yet these differences may lead to carcinogenesis.

Traditionally, cancer has been viewed as a disease caused by severe dysfunction of 
special cellular components and biological pathways, but its true origins are quite different (Kan et al., 2010). Cancer involves not merely fundamental cell processes such as death, proliferation, and differentiation but also all the biological processes of the cell (see Figure 2) (Cascante et al., 2010; Hoffman et al., 2011). Accordingly, Figure 3 shows that carcinogenesis involves not only extensively studied cellular components such as the cytoskeleton, mitochondrion, and cell membrane but also the emerging "foci" of endosomes and the endoplasmic reticulum (Cattaneo et al., 2011; Fry et al., 2011). Furthermore, cellular dysfunctions are exemplified by interactions between biological processes and cellular components, making cancer far more complicated than previous conceived. Explaining cancer, as elucidating life itself, requires greater efforts for breakthrough progress.

The understanding of carcinogenesis through a proteomics pathway approach is 1 way to simplify complicated cancer biology and discover therapeutic targets. In our study, the differentially expressed proteins were mainly located in metabolic pathways, ribosomes, spliceosomes, endocytosis, and otherpathways. Alterations in cellular metabolism are among the most consistent hallmarks of cancer (Sahin et al., 2011). To sustain rapid proliferation and counteract the hostile environment observed in tumors, cells must increase the rate of metabolic reactions to provide the ATP, lipids, nucleotides, and amino acids necessary for daughter cell production (Fulda et al., 2010). As shown in Table 4, 115 proteins were located in metabolic-related pathways. This fact alone stresses the importance of cancer metabolism research. Fortunately, therapies that target tumor metabolism are already being tested in preclinical and clinical studies. Inhibition of pyruvate kinase isoenzyme M2 by small hairpin RNA significantly decreases ATP synthesis and inhibits tumor growth both in vivo and in vitro (Shi et al., 2010). Ribosome biogenesis as a possible contribution to cancer and potential therapeutic target for cancer treatment has been widely investigated (Montanaro et al., 2010). Some research has revealed that spliceosomes play important roles in cancer development, and targeting the SF3b subunit of spliceosomes with certain compounds has caused complete tumor remission in human tumor xenografts in vivo (Kotake et al., 2007; Fan et al., 2011). Accumulating evidence argues that many proteins can undergo nuclear translocation during endocytosis and affect gene expression directly. Although it is often unclear to what extent endocytic and nuclear functions are interrelated, several of these proteins are implicated in the regulation of cell proliferation and carcinogenesis, suggesting that their dual functions should be widely considered (Scita and Di Fiore, 2010). Aforementioned are several representative altered pathways in our research. Undoubtedly, these pathways deserve further study and could potentially be used as therapeutic targets.

The elucidation of the internal interactions between neoplastic cells and stromal cells and the development of network-based multi-target strategies are urgently needed to improve therapeutic efficacy (Eng et al., 2009). In our study, interactive networks between cancer and stromal cells were assumed to be mediated through repeatedly expressed proteins in cancer/ cancer stromal cells. After comparison, 186 proteins supported this hypothesis and are thought to play important roles in tumor-stroma interactions. As shown in Tables 2 and 5, most of these proteins have previously been correlated with cancer and are located in well-known biological pathways. These data present further evidence that epithelial cancer should be evaluated as a functional and complex system along with stromal components. One hundred and thirty-nine of the 186 proteins with specifically repeated expression in cancer/cancer stromal tissue are located in the biological KEGG pathways and play important roles in spliceosomes, focal 
adhesion, extracellular matrix-receptor interaction, oxidative phosphorylation, and other processes. These proteins can be ideal biomarkers for double molecular-targeted therapy. Based on the concept of tumor-stroma interaction, targeting blockades of repeatedly expressed proteins in biological pathways in cancer and stroma can influence the same signaling pathway in both neoplastic cells and stromal cells, thereby improving the capability to inhibit tumor progression and growth without killing normal tissue. One example is the pathway of oxidative phosphorylation, which maintains not only the primary function of cells but also the "hotspots" in cancer research. A new form of stromal-epithelial metabolic coupling is now well accepted - namely, that stromal cells fuel cancer cells by producing lactate or pyruvate and directly increase ATP production. This new parasitic form is called the reverse Warburg effect (Martinez-Outschoorn et al., 2011).

As listed in Table 5, specifically repeatedly expressed proteins in cancer tissue located in oxidative phosphorylation pathways indicate parallel metabolic changes in both tumor and surrounding stroma. This fact offers further evidence of metabolic coupling. Meanwhile, targeting these proteins may simultaneously block energy generation and transport in both tumor and stroma, breaking stromal-epithelial metabolic coupling and amplifying therapeutic efficacy. Although the aforementioned mechanism requires validation, we have reasons to believe that the proteins listed in Table 5 should be studied thoroughly for targeted therapy.

In the present study, we identified 1753 proteins that are differentially expressed in cancer and normal tissues. Most of these proteins have not been previously linked to muscleinvasive BTCC. GO enrichment/depletion analysis indicated cancer involving all of the biological processes as well as all cellular components. Five hundred and eighty-six differential proteins are in the well-known biological KEGG pathways, including metabolic pathways, spliceosomes, endocytosis, oxidative phosphorylation, and others. One hundred and thirty-nine of the 186 proteins with specifically repeated expression in cancer tissue are located in the biological KEGG pathways and are thought to be candidate biomarkers for multi-target therapy.

\section{ACKNOWLEDGMENTS}

Research supported by a grant from the National Natural Science Foundation of China (\#30901481) and the Doctoral Science Foundation of Shandong Province, China (\#BS2010YY009).

\section{REFERENCES}

Cascante M, Benito A, Zanuy M, Vizán P, et al. (2010). Metabolic network adaptations in cancer as targets for novel therapies. Biochem. Soc. Trans. 38: 1302-1306.

Cattaneo M, Lotti LV, Martino S, Alessio M, et al. (2011). Secretion of novel SEL1L endogenous variants is promoted by ER stress/UPR via endosomes and shed vesicles in human cancer cells. PLoS One 6: e17206.

Eng C, Leone G, Orloff MS and Ostrowski MC (2009). Genomic alterations in tumor stroma. Cancer Res. 69: 6759-6764.

Fan L, Lagisetti C, Edwards CC, Webb TR, et al. (2011). Sudemycins, novel small molecule analogues of FR901464, induce alternative gene splicing. ACS Chem. Biol. 6: 582-589.

Fry WH, Simion C, Sweeney C and Carraway KL, III (2011). Quantity control of the ErbB3 receptor tyrosine kinase at the endoplasmic reticulum. Mol. Cell Biol. 31: 3009-3018.

Fulda S, Galluzzi L and Kroemer G (2010). Targeting mitochondria for cancer therapy. Nat. Rev. Drug Discov. 9: $447-464$.

Hoffman BD, Grashoff C and Schwartz MA (2011). Dynamic molecular processes mediate cellular mechanotransduction. Nature 475: 316-323.

Jemal A, Siegel R, Xu J and Ward E (2010). Cancer statistics, 2010. CA Cancer J. Clin. 60: 277-300.

Genetics and Molecular Research 12 (4): 4251-4263 (2013)

CFUNPEC-RP www.funpecrp.com.br 
Kan Z, Jaiswal BS, Stinson J, Janakiraman V, et al. (2010). Diverse somatic mutation patterns and pathway alterations in human cancers. Nature 466: 869-873.

Kaufman DS, Shipley WU and Feldman AS (2009). Bladder cancer. Lancet 374: 239-249.

Kotake Y, Sagane K, Owa T, Mimori-Kiyosue Y, et al. (2007). Splicing factor SF3b as a target of the antitumor natural product pladienolide. Nat. Chem. Biol. 3: 570-575.

Li D, Li JQ, Yang SG, Wu SF, et al. (2005). An integrated strategy for functional analysis in large-scale proteomic research by gene ontology. Prog. Biochem. Biophys. 32: 1026-1029.

Martinez-Outschoorn UE, Pavlides S, Howell A, Pestell RG, et al. (2011). Stromal-epithelial metabolic coupling in cancer: integrating autophagy and metabolism in the tumor microenvironment. Int. J. Biochem. Cell Biol. 43: 1045-1051.

Montanaro L, Treré D and Derenzini M (2008). Nucleolus, ribosomes, and cancer. Am. J. Pathol. 173: 301-310.

Niu HT, Xu T, Zhang YB, Pang DQ, et al. (2008). Outcomes for a large series of radical cystectomies for bladder cancer. Eur. J. Surg. Oncol. 34: 911-915.

Pezaro C, Liew MS and Davis ID (2012). Urothelial cancers: using biology to improve outcomes. Expert. Rev. Anticancer Ther. 12: 87-98.

Roth LM and Czernobilsky B (2011). Perspectives on pure ovarian stromal neoplasms and tumor-like proliferations of the ovarian stroma. Am. J. Surg. Pathol. 35: e15-e33.

Sahin E, Colla S, Liesa M, Moslehi J, et al. (2011). Telomere dysfunction induces metabolic and mitochondrial compromise. Nature 470: 359-365.

Sakamoto S, McCann RO, Dhir R and Kyprianou N (2010). Talin1 promotes tumor invasion and metastasis via focal adhesion signaling and anoikis resistance. Cancer Res. 70: 1885-1895.

Schiffer E, Vlahou A, Petrolekas A, Stravodimos K, et al. (2009). Prediction of muscle-invasive bladder cancer using urinary proteomics. Clin. Cancer Res. 15: 4935-4943.

Scita G and Di Fiore PP (2010). The endocytic matrix. Nature 463: 464-473.

Shao C, Liu Y, Ruan H, Li Y, et al. (2010). Shotgun proteomics analysis of hibernating arctic ground squirrels. Mol. Cell Proteomics 9: 313-326.

Shi HS, Li D, Zhang J, Wang YS, et al. (2010). Silencing of pkm2 increases the efficacy of docetaxel in human lung cancer xenografts in mice. Cancer Sci. 101: 1447-1453.

Thomas S, Overdevest JB, Nitz MD, Williams PD, et al. (2011). Src and caveolin-1 reciprocally regulate metastasis via a common downstream signaling pathway in bladder cancer. Cancer Res. 71: 832-841.

Tong W, Harris S, Cao X, Fang H, et al. (2004). Development of public toxicogenomics software for microarray data management and analysis. Mutat. Res. 549: 241-253.

Wooster RF (2012). Tumor-stroma relationships: who's the driver? Cell Cycle 11: 1753.

Yamazaki K, Takamura M, Masugi Y, Mori T, et al. (2009). Adenylate cyclase-associated protein 1 overexpressed in pancreatic cancers is involved in cancer cell motility. Lab. Invest. 89: 425-432. 